\title{
Neuronal Activity in Substantia Nigra Pars Reticulata during Target Selection
}

\author{
Michele A. Basso ${ }^{1}$ and Robert H. Wurtz ${ }^{2}$ \\ ${ }^{1}$ Department of Physiology, University of Wisconsin-Madison, Madison, Wisconsin 53706, and 'Laboratory of \\ Sensorimotor Research, National Eye Institute, Bethesda, Maryland 20892
}

\begin{abstract}
Complex visual scenes require that a target for an impending saccadic eye movement be selected from a number of possible targets. We investigated whether changing the number of stimuli from which a target would be identified altered the activity of substantia nigra pars reticulata $(\mathrm{SNr})$ neurons of the basal ganglia (BG) and how such changes might contribute to changes we observed previously in the superior colliculus (SC). One, two, four, or eight visual stimuli appeared on random trials while monkeys fixated a centrally located spot. After a delay, one of the stimuli in the array changed luminance, indicating that it was the saccade target. We found that $\mathrm{SNr}$ neurons that had a pause in tonic activity after target onset and when the saccade was made to the target showed a modulation of activity during the multitarget task. Because the number of stimuli in the array increased from one to eight, the initial pause after the onset of the visual stimulus decreased. Activity during the preselection delay was reduced but was independent of the
\end{abstract}

Visual scenes encountered during natural viewing require that a single stimulus be selected out of many possible to serve as an object directing visual attention or as a target identifying a goal for actions. Voluntary saccadic eye movements, those rapid reorienting movements of the eye, are an elegant model behavior in which to study target selection for action, because goal directed movements, by definition, require the identification of a single goal.

Our previous work examined the role of intermediate layer superior colliculus (SC) neurons in a task in which one target of many possible was identified for a saccadic eye movement. We found that the activity of SC neurons reflected the probability that a particular saccade target would be selected for a saccade (Basso and Wurtz, 1998). Similar reports have been described for these neurons in gap saccade tasks (Dorris and Munoz, 1998). Therefore, some SC neuronal activity is not obligately linked to the production of a saccade and therefore may reflect processes intervening between vision and action (Glimcher and Sparks, 1992; Munoz and Wurtz, 1995).

Because the basal ganglia $(\mathrm{BG})$ receive direct input from multiple cortical regions reflecting target selection as well as areas

\footnotetext{
Received Oct. 9, 2001; revised Dec. 17, 2001; accepted Dec. 18, 2001.

We are gratef ul to Dr. John McClurkin for data analysis software, the Laboratory of Diagnostic Radiology at National Institutes of Health for providing magnetic resonance images, Dr. Harvey Karten and Agnieska Brzozska-Precthl for immunohistochemical processing of the tissue, and Jennifer Pokorny for her assistance during the preparation of this manuscript.

Correspondence should be addressed to Dr. Michele A. Basso, Department of Physiology, University of Wisconsin, Madison, Medical School, 1300 University Avenue, Room 291 MSC, Madison, WI 53706. E-mail: michele@physiology. wisc.edu.

Copyright (ㄷ) 2002 Society for Neuroscience $\quad 0270-6474 / 02 / 221883-12 \$ 15.00 / 0$
}

number of possible targets present. When one of the stimuli was identified as the saccade target, but before the saccade was made, we found a sharp decline in activity. This decline was related to the monkey's selecting the target rather than the luminance change identifying the target, because on error trials, when the luminance changed but a saccade was not made to the target, the activity did not decline. The decline for the preferred target location was also accompanied by a lesser decline for adjacent locations. Our findings indicate that $\mathrm{SNr}$ activity changes with target selection as it does with saccade initiation and that the SNr could make substantial, direct contributions to the SC at both times. The pause in SNr activity with target selection is consistent with the hypothesis that BG provide a disinhibition for the selection of desired movements.

Key words: saccade; primate; inhibition; disinhibition; competition; vision

modulated by visual attention (Selemon and Goldman-Rakic, 1985, 1988; Boussaoud et al., 1992; Parthasarathy et al., 1992), and the substantia nigra pars reticulata $(\mathrm{SNr})$, one of two output nuclei of the BG, has direct projections to the SC (Hopkins and Niessen, 1976; Anderson and Yoshida, 1977; Deniau et al., 1978; Graybiel, 1978; Beckstead, 1983; Hikosaka and Wurtz, 1983d; Karabelas and Moschovakis, 1985), in the current experiment we hypothesized that we would see activity modulation in the $\mathrm{SNr}$ reflecting changes in target probability. Changes in SNr activity might occur under the same conditions as those seen in SC and therefore might be regarded as precursors to the SC changes that were related to target selection.

Recent experimental work has inspired a renewed consideration of the role of BG nuclei in events other than movement initiation. For example, anatomical (Hazrati and Parent, 1992a,b; Parent and Hazrati, 1993) and physiological data (Mink and Thatch, 1991a,b) suggest that BG are involved in selecting preferred movements through disinhibition and suppressing activity associated with nonpreferred movements through inhibitory mechanisms (Mink and Thatch, 1993; Mink, 1996). Indeed, that SNr neurons pause for saccadic eye movements is strong support for the role of disinhibition from the $\mathrm{BG}$ in movement initiation (Hikosaka and Wurtz, 1983a,c,d; Handel and Glimcher, 1999, 2000). We reasoned that we could address some of the issues related to events before saccade initiation in the $\mathrm{SNr}$, as we had done previously in the SC. Therefore, in the present work, we recorded $\mathrm{SNr}$ neurons while subjects performed the same behavioral task that we used previously to measure SC neuronal activity. 
Three observations from our previous work (Basso and Wurtz, 1998) are relevant to the present experiment. First, as the number of possible targets increases, the activity of SC neurons decreases, which is evidence of lateral interactions. Second, when a single target is identified out of the many possible, the activity of SC neurons increases to levels seen when only a single target is present, overcoming the inhibitory interactions and analogous to changes seen in cortical regions when visual attention is directed to the preferred target. Third, when monkeys make saccades to targets located adjacent to and opposite the preferred response field, the activity of some SC neurons is suppressed. This finding is similar to that seen in the frontal eye field (Schall and Hanes, 1993; Schall, 1995; Schall et al., 1995) and may reflect a mechanism of target selection for saccades.

By examining the activity of SNr neurons as we did with SC and comparing the results with those that we obtained previously in the SC, we could determine whether $\mathrm{SNr}$ activity modulations could be directly responsible for the expression of SC activity modulations. We found that $\mathrm{SNr}$ neurons were modulated by changes in the probability that a particular stimulus would become the target for the saccade, consistent with a role for these neurons in target selection for saccades. However, given the nature of the modulations in $\mathrm{SNr}$ seen with changes in target probability, additional modulations must be responsible for the modulation of SC with changes in target probability. In sum, the $\mathrm{SNr}$ activity changes are consistent with, and provide constraints on, recent views of BG function and behavioral selection.

\section{MATERIALS AND METHODS}

Physiological procedures. Two monkeys were prepared for chronic electrophysiological recording of single neurons and eye movements. Anesthesia was induced initially with an intramuscular injection of ketamine $(10.0 \mathrm{mg} / \mathrm{kg})$, valium $(1.0 \mathrm{mg} / \mathrm{kg})$, and glycopyrrolate $(0.01 \mathrm{mg} / \mathrm{kg})$. Monkeys were intubated and maintained at a general anesthetic level with isofluorane. A subconjunctival eye coil was implanted (Judge et al., 1980). A plastic head holder for restraint and a cylinder for microelectrode recording were mounted on the top of the exposed skull and secured with titanium screws and dental acrylic. This hardware allowed subsequent magnetic resonance images to be obtained. For access to the $\mathrm{SC}$, the recording chamber was placed stereotaxically on the midline and angled $38^{\circ}$ back so that the electrode penetrations were directed caudorostral, toward the SC. For the SNr, two cylinders were placed on the skull. The cylinder on the left side was angled $30^{\circ}$ lateral-medial at stereotaxic coordinates, anteroposterior (AP) 9.0 and mediolateral (ML) -5.0 (O. Hikosaka, personal communication). The second cylinder was placed on the right side at stereotaxic coordinates AP 9.0 and ML 5.0, parallel to the surface of the skull, i.e., a $0^{\circ}$ angle. This approach allowed straight vertical penetrations through the ventrobasal complex of the thalamus (VPM) and provided recording of the trigeminal recipient neurons of the thalamus as a landmark (A. Handel, P. Glimcher, and W. Schultz, personal communication). Figure 2 shows coronal sections through the $\mathrm{SNr}$ of one of these monkeys with a $0^{\circ}$ penetration. At the end of surgery and $1 \mathrm{~d}$ after the operation, animals were given Banamine for analgesia. An antibiotic (Polyflex) was given $1 \mathrm{~d}$ before the operation and every other day for $14 \mathrm{~d}$ after the operation. Monkeys recovered for 1 week before behavioral and physiological recording commenced. All protocols were approved by the Institute Animal Care and Use Committee and complied with the Public Health Service Policy on the humane care and use of laboratory animals.

Single neurons were recorded with tungsten microelectrodes (Frederick Haer) with impedances between 0.7 and $1.5 \mathrm{M} \Omega$ measured at $1 \mathrm{kHz}$. Electrodes were aimed toward the recording site through stainless steel guide tubes held in place by a delrin grid that was secured to the recording chamber (Crist et al., 1988). Action potential waveforms from individual neurons were identified with a window discriminator that returned a transistor-transistor logic (TTL) pulse for each waveform that met both time and amplitude criteria. The time of occurrence of each action potential was stored with $1 \mathrm{msec}$ resolution. To identify the $\mathrm{SNr}$ before the experiment, we searched for neurons that were antidromically activated by stimulation within the SC (Hikosaka and Wurtz, 1983d). For this, electric current was passed through tungsten microelectrodes (Frederick Haer) with impedances between 0.1 and $0.7 \mathrm{M} \Omega$ measured at 1 $\mathrm{kHz}$. Single, biphasic pulses $150 \mu \mathrm{sec}$ in duration were used. Current intensities varied with the searching currents ranging between 200 and $1000 \mu \mathrm{A}$. Antidromic currents varied between 50 and $400 \mu \mathrm{A}$ (Lemon, 1984). Neurons identified as antidromic had a short $(<2.0 \mathrm{msec})$ and consistent latency and were also subjected to the collision test (Fuller and Schlag, 1976). For this, the TTL pulse derived from a spontaneously occurring SNr spike was provided as input to a stimulator (Astro-Med Instruments S88) providing a current-balanced pulse to the SC. This test was inconclusive in some cases because of the short latency of the $\mathrm{SNr}$ antidromic spike and the delays inherent in the hardware used for the production of the TTL pulse (WEXv3.0a) (Chandra and Optican, 1997). At least one of both classes of neuron described in this report could be driven antidromically. The number of antidromic neurons was not large enough $(n=9)$ to draw conclusions about the projection pattern to the SC. Our purpose for using the antidromic technique was to confirm that our recordings were within the $\mathrm{SNr}$.

Behavioral procedures. All behavioral paradigms and storage of data were controlled by a 486PC running a QNX-based real-time data acquisition system (REX) (Hays et al., 1982). During experiments, monkeys were seated in an adjustable primate chair facing a screen with their heads restrained for the duration of the experiment (3-5 hr). The visual display on the screen was rear projected by a television projector (Liquid Crystal, Sharp) operating at $60 \mathrm{~Hz}$. The tangent screen was located $57 \mathrm{~cm}$ in front of the monkeys. The centrally located fixation point was a projected image of a light-emitting diode (LED). Eye movements were recorded with the magnetic search coil technique (Fuchs and Robinson, 1966), and horizontal and vertical eye position signals were sampled at 1 $\mathrm{kHz}$. An interactive computer program was used to make measurements and calculate metrics and dynamics of eye movements (DEX). Saccades were detected using velocity $\left(10-25^{\circ} / \mathrm{sec}\right)$ and acceleration $\left(500-800^{\circ}\right)$ $\mathrm{sec}^{2}$ ) criteria, and the data were inspected by the experimenter for corrections.

Neurons were identified while monkeys performed visually guided saccades or delayed saccades either memory guided (Hikosaka and Wurtz, 1983c) or visually guided. In the former task, a centrally located fixation point appeared, and the monkeys were required to maintain fixation of this spot within an electronic window of $2^{\circ}$. A peripherally located spot was presented for $200 \mathrm{msec}$ while the monkeys maintained fixation. After a delay of $200-800 \mathrm{msec}$ in which selection could occur, the fixation point was removed, and monkeys made a saccade to the location of the previously flashed target spot. In the later task, the target spot remained illuminated throughout the trial. For visually guided saccades, the fixation spot disappeared at the same time the target spot appeared, i.e., 0 msec delay. We determined the general characteristics of the neuronal activity and an estimate of the center of the preferred field by requiring monkeys to make saccades to different locations in the visual field. In general the fields of $\mathrm{SNr}$ neurons were large, in some cases encompassing an entire hemifield (Handel and Glimcher, 1999). We did not attempt to characterize the field fully. We made a qualitative assessment on-line about the preferred location on the basis of the largest decline in activity. During all experiments, monkeys were rewarded with a drop of fruit juice or water. Monkeys worked daily until satiated and were given supplemental fluid as required. The monkeys' weight was monitored daily, and they remained under the supervision of the institute veterinarian.

Target probability task. To determine the effect of target probability on $\mathrm{SNr}$ neuronal activity, we used the same multitarget task that we used previously for recordings in the SC (Basso and Wurtz, 1998). The details of this task are described below.

In the multitarget task (Fig. 1), our goal was to separate the sequence of events leading up to saccade generation while varying the probability that a given stimulus would become a saccade target. First, a centrally located fixation point (LED) was illuminated, and monkeys were required to look at it for $1 \mathrm{sec}$ to initiate the trial. Second, one, two, four, or eight spots of light were projected for a randomized time ranging from 800 to $1200 \mathrm{msec}$, and these trial types were randomly interleaved. This was the period of preselection because the monkeys did not know which of the spots would become the target. One of these possible targets was always located at the position in the visual field that yielded the maximal response of $\mathrm{SNr}$ neurons, in this case a maximal pause in activity. All other possible targets were placed equally eccentric but in different directions (in the four cardinal and four oblique directions, $45^{\circ}$ between 
Multitarget task

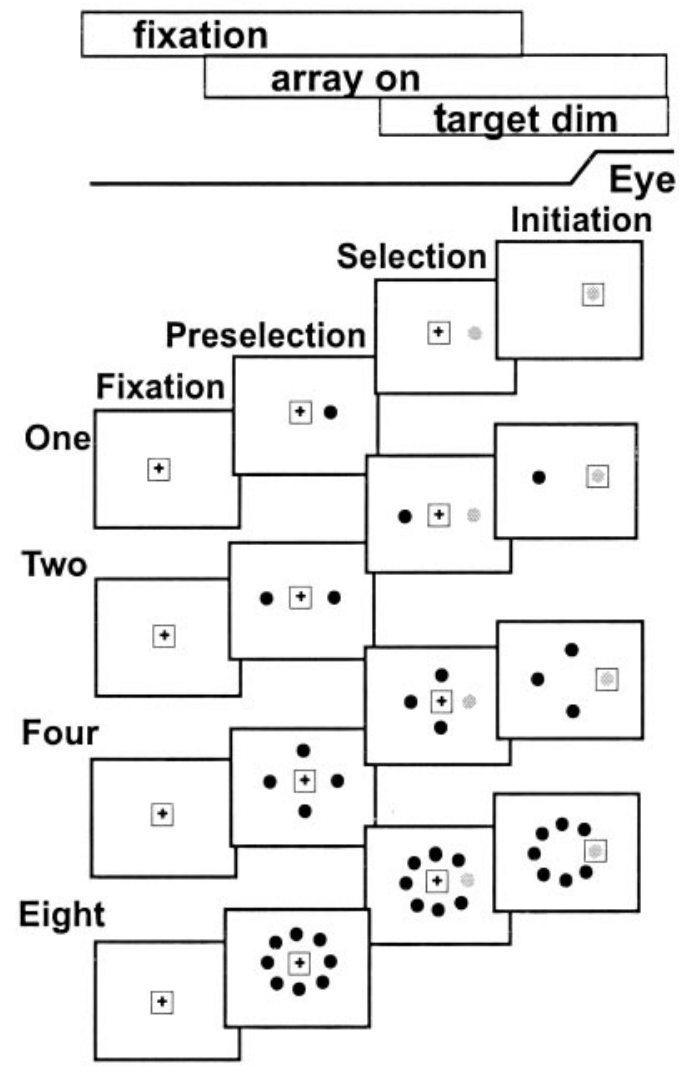

Figure 1. Multitarget task. Along the top, the bars labeled fixation, array on, and target dim depict the temporal sequence of the behavioral task used in this experiment. The line below, labeled Eye, is a schematic of eye position. The bottom portion of the figure depicts the spatial arrangement of the task and the different trial types. The large boxes are the screen on which visual stimuli were displayed. The cross represents the fixation point, and the small box indicates the eye position criterion window for correct performance of the task. Each of these trial types was randomly interleaved. As the number of possible target increased, the probability that any one would be identified for a saccade was decreased. The fixation period began with the onset of a fixation point located centrally on the screen. This was followed by a preselection period when the array of possible targets appeared. The selection period is indicated by the time in which the saccade target was identified by a reduction in luminance. The initiation period commenced when the cue to make a saccade, the removal of the fixation point, occurred. Each period of the task was separated by a random interval.

each of eight stimuli). The eccentricities ranged from 5 to $25^{\circ}$, with most being between 10 and $15^{\circ}$. Third, one of the possible targets dimmed for $800-1200$ msec. We defined this as the period of selection because the dimming indicated which of the spots was the target for the saccade. The final period of saccade initiation began when the fixation point went off (go signal), which required the monkeys to make a saccade within 500 msec to the dimmed target. Monkeys were required to maintain their eye position at the target for $300-500 \mathrm{msec}$ to obtain liquid reward. The data that we present are taken from trials in which the monkeys performed the task correctly and made saccades to the target located within the center of the preferred field, unless stated otherwise. The task had a clear target change so that it was essentially a pop-out task (Bravo and Nakayama, 1992) requiring only target detection, not discrimination (Treisman and Gelade, 1980).

Data analysis. In addition to descriptive statistics, we used parametric statistical procedures, provided the initial tests of normality were successful. When we compared more than two levels of a variable, we used the ANOVA. When we compared only two levels of a variable, we used $t$ tests. For multiple group comparisons, such as those in the multitarget task, we used repeated measures ANOVA with the Tukey test method

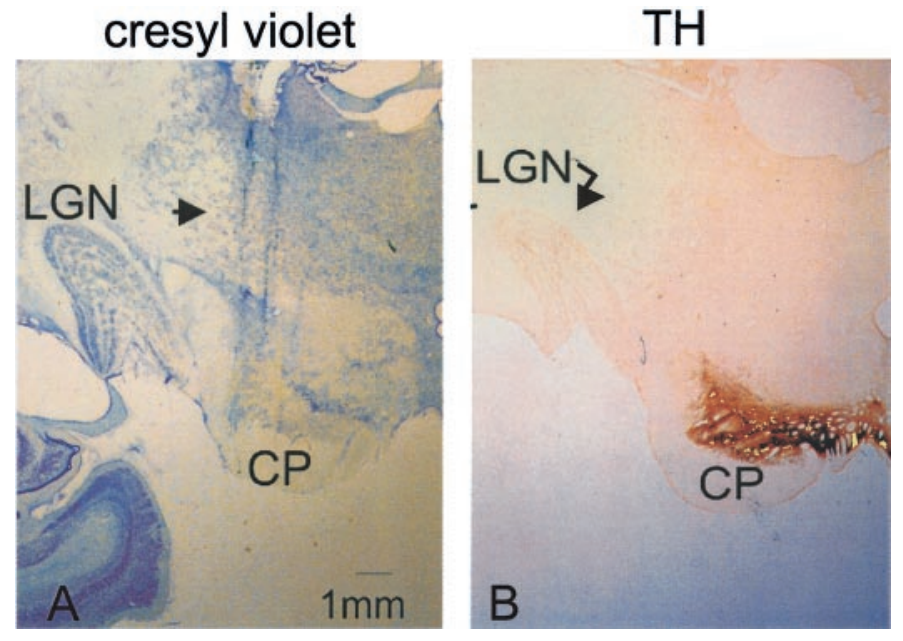

Figure 2. The $\mathrm{SNr}$ and $\mathrm{SNc}$ of a rhesus monkey. Two adjacent $(50 \mu \mathrm{m})$ coronal sections are presented. In $A$, the section was stained with cresyl violet and the electrode path is evident (arrow). In $B$, the adjacent section was stained immunohistochemically for tyrosine hydroxylase $(T H)$, the rate-limiting enzyme in the synthesis of the neurotransmitter dopamine (Cooper et al., 1986). The brown reaction product indicates the presence of $\mathrm{TH}$. These sections demonstrate that the electrode penetrations made in these experiments passed through the $\mathrm{SNc}$ and into the $\mathrm{SNr}$. CP, Cerebral peduncle; $L G N$, lateral geniculate nucleus.

for post hoc pairwise comparisons. If normality tests failed, the nonparametric equivalent of these tests was used. Comparisons generally involved measuring the mean level of discharge for the neurons in the one, two, four, and eight possible target conditions. We did this separately for successive time intervals in the task. For example, to analyze the visual response of neurons in the task, we performed a one-way ANOVA with four levels, namely, one possible target condition, two possible targets condition, four possible targets condition, and eight possible targets condition. When a main effect was obtained, we performed the pairwise comparisons using the Tukey test to determine which pair contributed to the significant difference.

\section{RESULTS}

\section{Neuronal classes}

We recorded from 81 neurons in two monkeys in four hemispheres. Of the 81 neurons, 58 had activity related to visual stimuli or saccades or both and will be the focus of the present report. In addition to using antidromic activation (Materials and Methods), we identified the SNr (Fig. 2) by observing the activity profiles of the neurons while monkeys performed the visually guided saccade task and the delayed saccade task (either visually guided or memory guided). We classified the neurons according to their activity in three periods during performance of a delayed saccade task to the preferred target location (or in the single target condition of our multitarget task if no delayed saccade task was performed): baseline period, $200 \mathrm{msec}$ before visual stimulus onset while the subject actively fixated; visual period, 100-300 msec after visual stimulus onset; and saccade period, $150 \mathrm{msec}$ before and after saccade onset. We identified two types of $\mathrm{SNr}$ neurons (Fig. 3). "Visual-saccade" neurons had a decline of at least $1 \mathrm{SD}$ below baseline during either the visual or both the visual and the saccade intervals for a least $15 \%$ of the trials $(n=$ 38). "Saccade" neurons had a decline in activity for at least 1 SD below baseline for at least $15 \%$ of the trials only during the saccade interval $(n=20)$.

For the other 23 of the 81 neurons, 2 were recorded from the zona incerta as indicated by their tonic firing rate and their omnidirectional pause in activity associated with the onset of 


\section{Delayed Saccade Task}

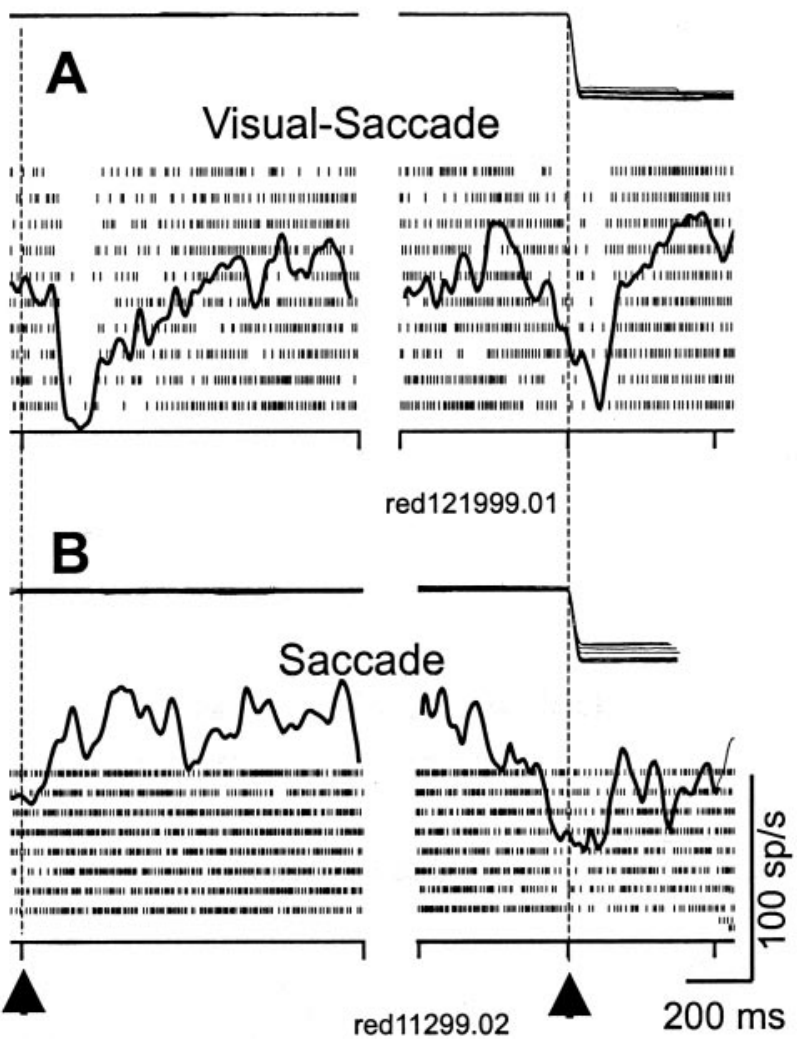

Figure 3. Examples of neuronal activity profiles in the SNr. The left column is aligned on the onset of the visual target (vertical dashed line and arrow), and the right column is aligned on the saccade (vertical dashed line and arrow). Eye position traces are plotted, superimposed as a function of time. Individual ticks are action potentials, and each row of ticks is a trial. Spike density functions are superimposed on the raster diagram. The spike density functions were calculated with a Gaussian of $12 \mathrm{msec} . A, \mathrm{SNr}$ visual-saccade neurons have a pause for the onset of the visual stimulus as well as before the saccade. $B, \mathrm{SNr}$ saccade neurons show a decline in activity at the time of the saccade.

visually guided saccades (Ma, 1996) and spontaneous saccades (Hikosaka and Wurtz, 1983a). Thirteen neurons recorded were not related to saccades or to visual stimuli and were not modulated in our task. Eight neurons showed pauses in activity with the onset of the fixation point as described by Hikosaka and Wurtz (1983b). In our task, these neurons reduced their activity at the time of fixation and remained at that level throughout the duration of the trials independent of the number of stimuli. These 23 neurons will not be considered further.

\section{Multiple stimulus interactions}

\section{Visual-saccade neurons}

The $38 \mathrm{SNr}$ visual-saccade neurons had tonic spontaneous activity that showed systematic decreases throughout the trial in the multitarget task, and these sequential changes are best illustrated in the one target condition of this task (Fig. 4, One). After the stimulus appeared, the activity decreased (Fig. 4, One, left column). This decreased activity recovered to a tonic level later in the preselection delay period, but this level was less than the spontaneous level (Fig. 4, One, left column). At the time the target dimmed at the beginning of the selection delay period, the neuronal activity declined precipitously (Fig. 4, One, middle column, vertical dashed line). This decline in activity was maintained until

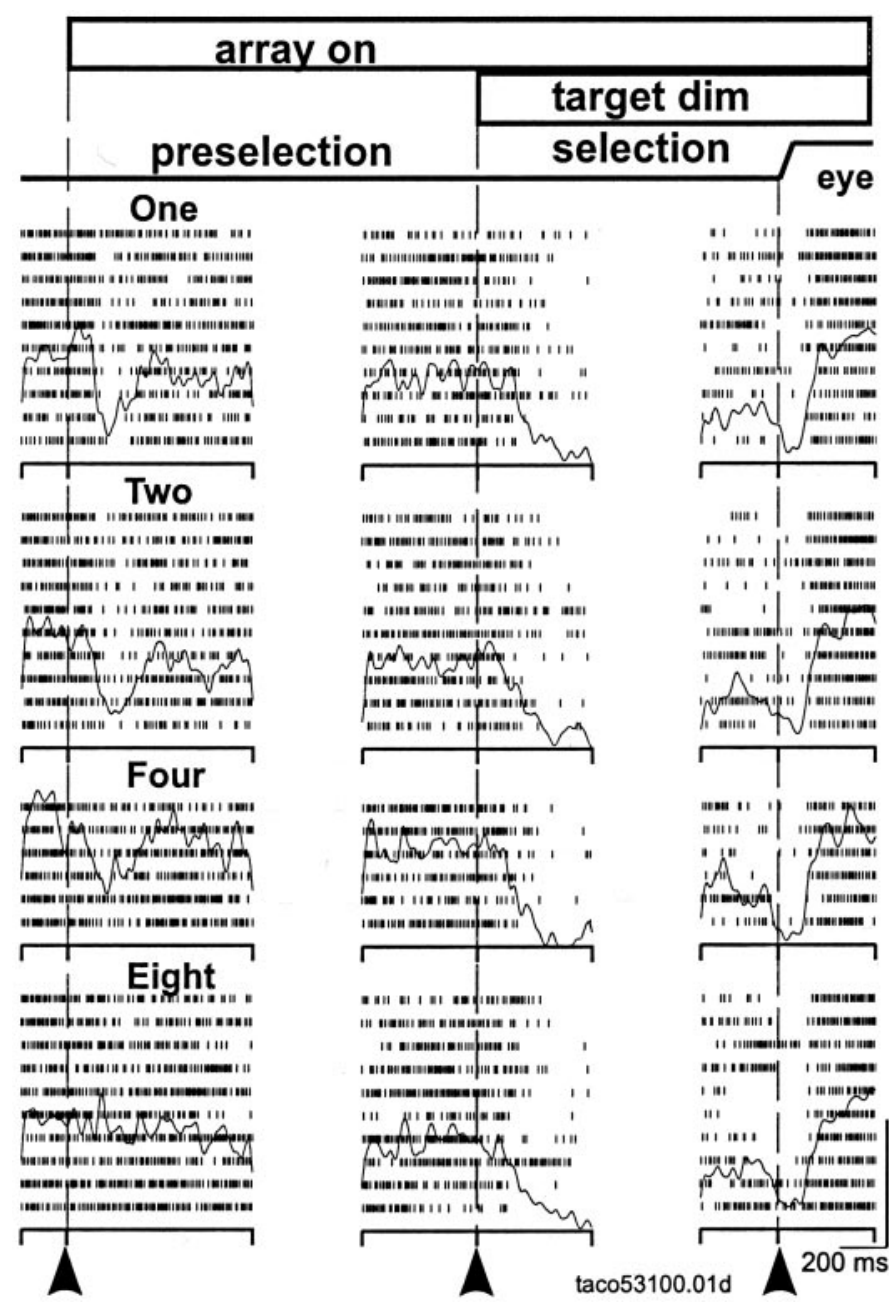

Figure 4. Effect of changes in target probability on an $\mathrm{SNr}$ visual-saccade neuron. The events of the task are indicated as the labeled periods across the top. The eye position trace is a schematic. The first row of rasters is for correct responses in the single possible target condition (One), the second is when two possible targets appeared (Two), and the third is when four possible targets appeared (Four). The last row is when eight possible targets appeared (Eight). The columns of rasters are aligned on the events of the task: the first is aligned on when the stimuli appear, the second is aligned on target identification, by dimming, and the last is aligned on saccade initiation. Each tick in the raster is a single action potential, and each row of ticks is an individual trial. The lines superimposed are spike density functions $(\sigma=12 \mathrm{msec})$. All data are taken from correct trials when the target was identified in the preferred field of the recorded neuron. The arrowheads and the vertical dashed lines indicate the trace alignment. The initial pause decreased with increasing numbers of possible targets, and at the time the target was identified (middle column, vertical dashed line), the activity dropped precipitously. Vertical calibration bar: 100 spikes/sec.

the saccade was initiated, showed a further dip with the saccade, and then eventually returned to baseline tonic levels shortly thereafter (Fig. 4, One, right column). Use of the multitarget task shows that in addition to the well established decreases in activity related to visual target onset and saccade initiation, these $\mathrm{SNr}$ neurons showed both a tonic depression of activity in the preselection period and a sharp decline in activity when the instruction to make the saccade was given at the start of the selection period.

As the number of possible targets increased, the pause associated with the onset of the stimulus array decreased, and this pause was absent in the presence of eight possible targets (Fig. 4, 


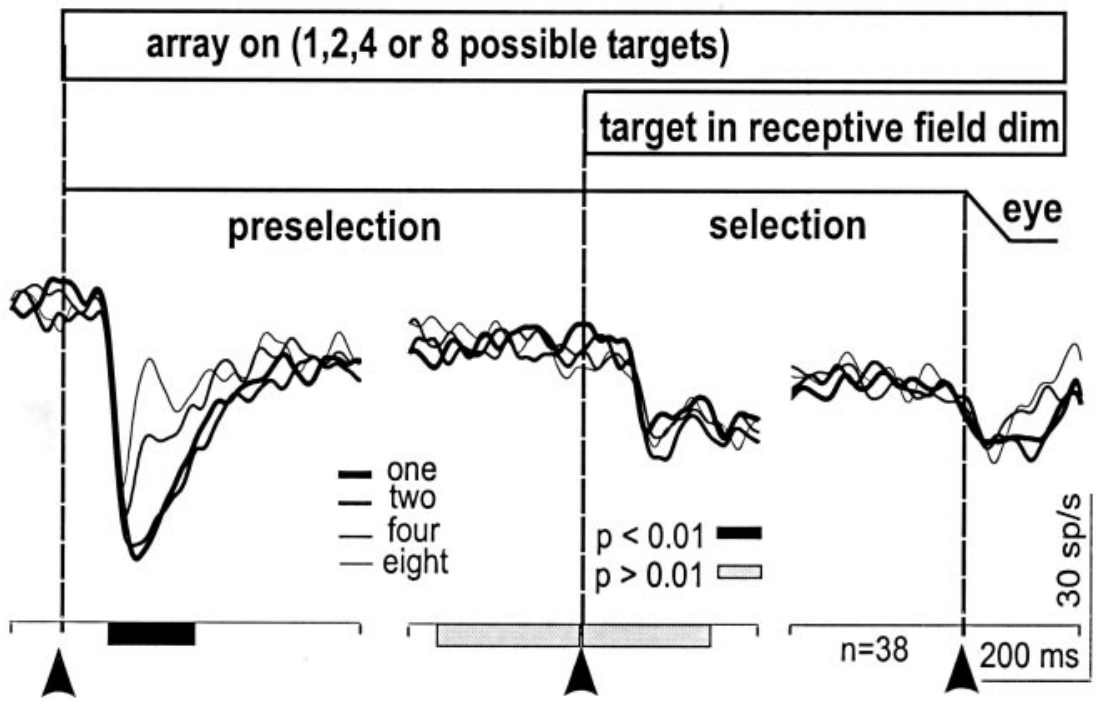

Figure 5. The activity across the sample of visualsaccade $\mathrm{SNr}$ neurons was modulated with changes in target probability. The traces show the mean spike density function of $38 \mathrm{SNr}$ neurons in each target probability condition. For these traces, the target was always located in the center of the response field, and the trials were performed correctly. The black bars indicate a statistically significant difference between the four conditions during the measurement intervals. The gray bars indicate a lack of statistical significance. The initial preselection measurement interval was from 100 to $400 \mathrm{msec}$ after the stimuli appeared. The second preselection interval was $400 \mathrm{msec}$ before the target was identified. Because there was a minimum of $800 \mathrm{msec}$ between task events, there is no overlap in the trace or the measurement intervals. The selection interval was $100-500 \mathrm{msec}$ after the target dimmed.

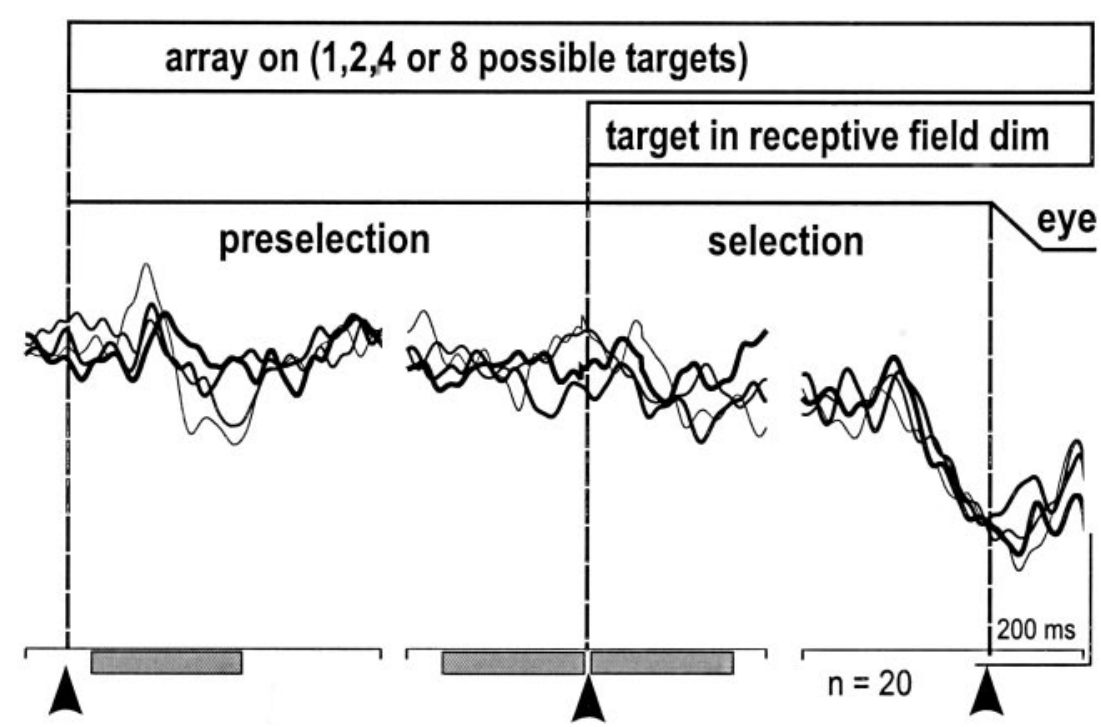

Figure 6. The activity across the sample of saccaderelated $\mathrm{SNr}$ neurons was not modulated with changes in target probability. The traces show the mean spike density function of $20 \mathrm{SNr}$ neurons in each target probability condition. The arrangement of this figure is identical to that in Figure 5. For these traces, the target was always located in the center of the response field, and the trials were performed correctly. The gray bars indicate a lack of statistical significance. The initial preselection measurement interval was from 100 to $400 \mathrm{msec}$ after the stimuli appeared. The second preselection interval was $400 \mathrm{msec}$ before the target was identified. Because there was a minimum of $800 \mathrm{msec}$ between task events, there is no overlap in the trace or the measurement intervals. The selection interval was $100-500 \mathrm{msec}$ after the target dimmed. Note that these neurons showed a clear decline in activity before the saccade was initiated. Vertical calibration: 20 spikes/sec.
Two, Four, Eight, left column). During the preselection delay period, the decreased tonic level of activity did not differ as the number of possible targets increased (Fig. 4, left side of middle column in One, Two, Four, Eight). At the time the target was identified, the decline in neuronal activity did not change as the number of possible targets increased (Fig. 4, dashed line, middle column). When the fixation point was removed, a saccade to the target was cued, and there was a modest, additional decline in activity at the time of the saccade. This saccade-related decline also was the same regardless of the number of stimuli (Fig. 4, vertical line, right column).

We averaged the neuronal activity from the sample of 38 visual-saccade neurons across the different periods of the task for each possible target condition and superimposed the averaged traces (Fig. 5). The pattern of activity across the sample was similar to the example neuron shown in Figure 4. As the number of possible targets increased, the pause in activity after the stimulus onset decreased (Fig. 5, left plot). The difference in the initial visual pause (50-200 msec after stimulus onset) in the different possible target conditions was statistically significant (ANOVA; $F_{(3,151)}=$ 20.09; $p<0.001$ ). Pairwise comparisons performed with the Tukey test revealed that all conditions contributed to the overall signifi- cance $(p<0.03)$ except the difference between possible targets conditions 1 and $2(p=0.97)$.

During the remainder of the preselection period, the activity was not significantly modulated with the number of possible targets $\left(\mathrm{ANOVA} ; F_{(3,303)}=0.18 ; p=0.95\right)$, but the activity that was measured $400 \mathrm{msec}$ before the target dimmed remained below the baseline level (200 msec before the array onset) across all conditions when differences among individual neurons were allowed (ANOVA; $F_{(1,303)}=158.39 ; p=0.001$ ) (Fig. 5, middle plot). At the time the selection period began, when the target dimmed (Fig. 5, middle plot, selection) the activity of the neurons declined dramatically, and the decline occurred similarly for each condition $(1,2,4$, and 8$)$. Comparison of the activity $400 \mathrm{msec}$ before the target dimmed and $400 \mathrm{msec}$ after the target dimmed for each possible target condition revealed significant differences $\left(\right.$ ANOVA; $\left.F_{(1,303)}=7.31 ; p<0.007\right)$. The amount of decline did not differ between the numbers of possible targets as indicated from the interaction term (ANOVA; $\left.F_{(3,303)}=0.13 ; p=0.94\right)$ (Fig. 5, middle plot, selection). At the time of and after saccade onset (Fig. 6, vertical dashed line in last set of traces), there was an additional decline in activity [see also Hikosaka and Wurtz (1983a)]. 
In summary, for the visual-saccade neurons, as the number of possible targets increases, the pause associated with the onset of the visual stimuli decreases. The activity in the preselection delay period remains below baseline but is independent of the number of possible targets. At the time the target becomes available for selection, the neuronal activity drops sharply, regardless of the number of visual stimuli present. The drop-off in activity remains until well after the saccade onset, at which point the neuronal activity returns to spontaneous levels.

\section{Saccade neurons}

The 20 saccade neurons had tonic, spontaneous activity that decreased only around saccade onset. In our multitarget task, the saccade neurons behaved differently from the visual-saccade neurons already described. In the single target condition, saccade neurons maintained a tonic rate of activity that was not different from the baseline level of firing and was maintained throughout the trial until slightly before saccade onset (Fig. 6, thickest black line). At the time of the saccade, there was a clear pause in activity that lasted the duration of the saccade and increased back to spontaneous levels at $\sim 300 \mathrm{msec}$ after the saccade (Fig. 6, selection). As the number of possible targets increased, the activity of saccade neurons across the sample was modulated slightly. With eight possible targets, the initial activity after stimulus onset increased (Fig. 6, thinnest black line) and then rapidly decreased, as if transiently oscillating. Nevertheless, the initial activity (50$200 \mathrm{msec}$ after stimulus onset; shaded gray bar on abscissa) was statistically indistinguishable across the four possible target conditions (ANOVA; $F_{(3,79)}=0.043 ; p=0.988$ ). To determine whether there were some differences obscured by the large window, we divided the initial response into three smaller windows: 75-125 msec after stimulus onset, 125-200 msec after stimulus onset, and 200-300 msec after stimulus onset. We performed ANOVAs on averaged spike counts during these intervals as well as on spike counts normalized to the baseline activity $(200 \mathrm{msec}$ before stimulus onset). The normalization was done individually for each neuron. In the first window the average activity in the single target condition was 79.57 spikes/sec, whereas with eight possible targets the average activity was 82.57 spikes/sec. The same trend was observed in the second window ( 84.26 vs 88.74 spikes/sec). In the third window, the opposite trend was revealed: 84 spikes/sec was the average rate for a single target, whereas 70.73 spikes/sec was the average rate with eight stimuli present. Statistical analyses revealed that none of these differences were statistically significant (ANOVA, $F_{(3,79)}=0.073, p=0.97,75-125$ msec window; ANOVA, $F_{(3,79)}=0.102, p=0.96,125-200 \mathrm{msec}$ window; ANOVA, $F_{(3,79)}=2.48, p<0.07,200-300 \mathrm{msec}$ window).

Comparison of the preselection period (400 msec before the target dimmed) to the period after a target was identified (400 msec after the target dimmed) revealed that the activity of saccade neurons did not change in any of the possible target conditions $\left(\mathrm{ANOVA} ; F_{(1,159)}=0.459 ; p=0.499\right)$. Additionally, there were no differences in saccade activity during the selection period for the different possible target conditions (ANOVA; $F_{(3,159)}=$ $0.052 ; p=0.985)$. Before the onset of the saccade, these neurons showed a pause in activity that lasted the duration of the saccade (Fig. 6, right plot). This pause did not differ for the different numbers of possible targets.

Thus, saccade-related neurons of the $\mathrm{SNr}$ are not consistently modulated with changes in the number of possible targets avail-

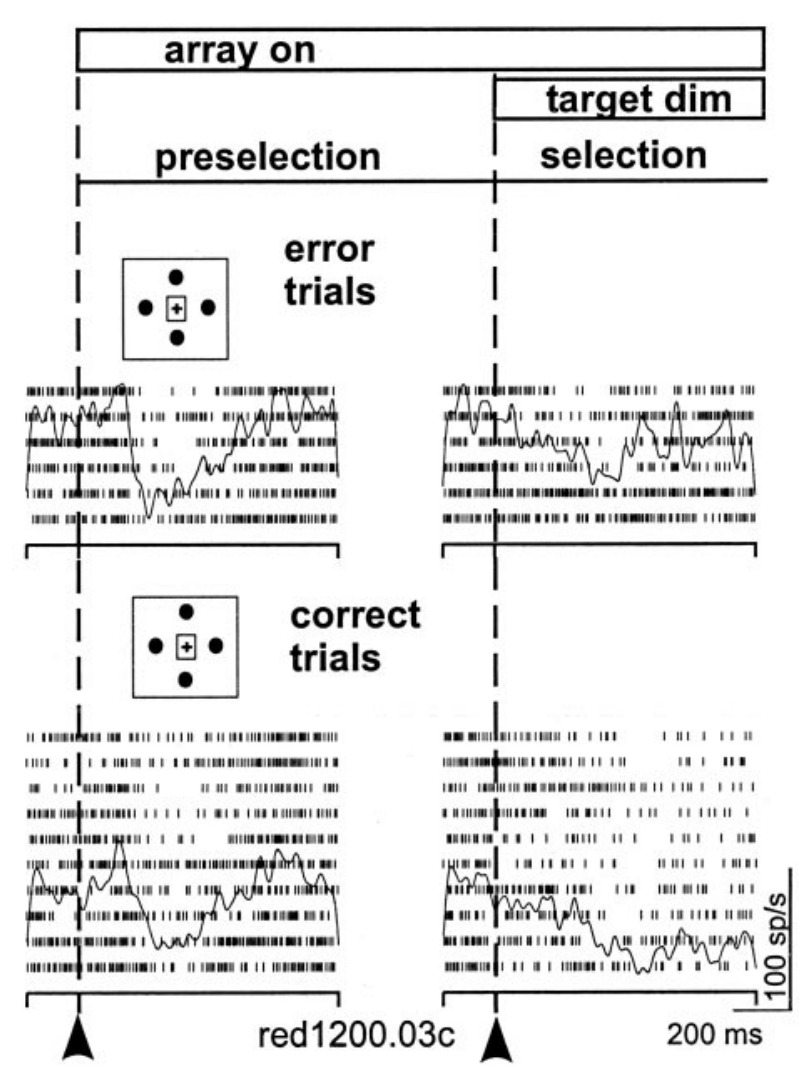

Figure 7. The activity decline of visual-saccade SNr neurons associated with target identification predicts the impending saccade. The temporal arrangement is shown on the top by the labeled bars. The vertical dashed lines indicate the alignment of the traces. The top raster and spike density functions are taken from trials in which four possible targets were presented and the monkey made an erroneous saccade to one of the other stimuli. The bottoms rasters and spike densities are taken from correct trials in the same four possible target conditions. Note that the time of saccade initiation is not indicated for clarity. The decline in $\mathrm{SNr}$ neurons associated with the dimming of one stimulus to indicate it as the target for a saccade does not occur if the monkey does not select the target as a goal for a saccade.

able for saccades or at the time the target became available for selection.

\section{Error trials}

Up to this point, we have considered only trials in which animals performed the task correctly; that is, saccades were made to the target that was identified during the selection period, and the saccades were made in a timely manner (within $500 \mathrm{msec}$ ) and were accurate within $2-3^{\circ}$. In some rare cases, however, monkeys made saccades to the wrong target or failed to make a saccade at all. This occurred infrequently because the task is simple and the monkeys were well trained, but their occurrence provided an opportunity to determine whether the change in SNr activity was related to the target presented or to the monkey's response to the target. Figure 7 shows the trials for a single visual-saccade neuron during trials with four possible targets. In some trials the monkey performed the task correctly (Fig. 7, bottom plot, correct trials), and in other trials the monkey made errors by making a saccade to another location (Fig. 7, top plot, error trials). Initially the neuron showed a pause associated with the onset of the visual stimulus array regardless of the trial outcome (Fig. 7, left plot). In correct trials, at the time the target dimmed, the activity of the 
neuron declined and remained low (Fig. 7, right plot). In contrast, in the error trials, the neuronal activity did not decline or did so slightly but returned quickly to its tonic level. This comparison indicated that the pause in $\mathrm{SNr}$ neurons occurring after the identification of the target but in advance of the saccadic eye movement predicts the intent of the monkey rather than being exclusively related to the stimulus change.

We confirmed this observation by examining all the error trials across all the data. We found 79 error trials (18 neurons) in which the monkey made a saccade to the wrong target or did not make a saccade at all. We measured the average activity in a $400 \mathrm{msec}$ interval beginning $200 \mathrm{msec}$ after the target dimmed in both correct and errors trials for these neurons. The median activity in errors trials was $79.61 \mathrm{spikes} / \mathrm{sec}$, whereas the median activity in correct trials was $46.25 \mathrm{spikes} / \mathrm{sec}$. This difference was statistically reliable as determined by a Wilcoxon signed rank test $(p<0.01)$. Thus, the pause in $\mathrm{SNr}$ neurons reflects the saccade target ultimately chosen by the monkeys rather than the visual stimulus on the retina.

\section{Spatial resolution of stimulus interactions Contralateral and ipsilateral targets}

A comparison of the neuronal activity during the conditions when targets are presented in the preferred field of the neuron and the field opposite allows an inference about the activity of neurons coding nonpreferred target locations, similar to inferences made in cortical neurons (Britten et al., 1992; Thompson et al., 1996), as well as those made by us in our previous work in the SC (Basso and Wurtz, 1998). Therefore, we compared the activity in the two and eight possible targets conditions when the target in the preferred location was identified and when the target in the opposite hemifield was identified. We averaged the activity of the sample of visual-saccade neurons across the intervals of the task and superimposed the traces for the ipsilateral and contralateral target locations for the two possible targets condition (Fig. 8A) and for the eight possible targets condition (Fig. $8 B$ ). For this comparison we used the data from correct trials from visual-saccade neurons.

For both the two stimulus and eight stimulus trials, the first difference between the activity of the SNr neurons with an ipsilateral target and a contralateral target occurred at the time the target was indicated (Fig. 8, middle plot, vertical dashed line). When the target was in the ipsilateral hemifield, there was no decline in the neuronal activity (Fig. 8, vertical dashed line). The pause in activity was specific for the target at the preferred location in the contralateral field, and the change was significant $t_{(74)}=2.70, p<0.009$ for two stimuli and $t_{(74)}=2.76, p<0.007$ for eight stimuli). As the selection period continued, the neuronal activity remained below baseline (Fig. 8, rightmost plot). At the time of the saccade, however, the neuronal activity declined only for the saccade made to the target at the preferred location.

In summary, the decline in $\mathrm{SNr}$ activity around the time a target is identified and a saccade is made does not occur when the target is placed symmetrically in the ipsilateral visual field. The neurons coding the symmetrical nonpreferred target are primarily unaffected by the selection process in this task. The specificity of the decline also indicates that it is associated with the preferred field of the neuron rather than with any general arousal effect. Moreover, a lack of increase when the opposite hemifield target is identified suggests that there is not a simple, push-pull interaction between the two SNrs.
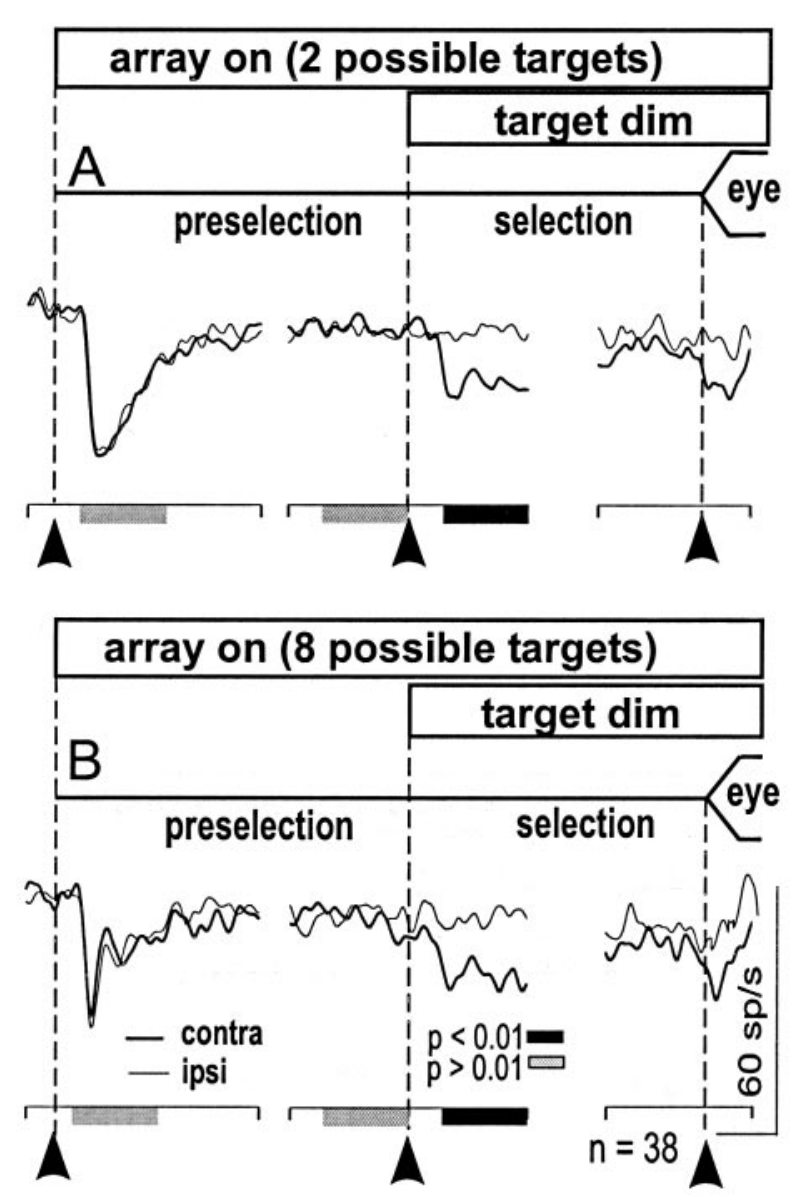

Figure 8. Comparison of neuronal activity related to identification of a target in and out of the preferred field of visual-saccade $\mathrm{SNr}$ neurons. The plot shows the mean activity of $38 \mathrm{SNr}$ neurons. The arrangement of this plot is the same as in Figures 5 and 6. $A$ shows the two possible targets condition when the target was identified in the preferred field (contra, thick line) and when the target was identified in the opposite hemifield (ipsi, thin line). The black bars indicate that there was a statistically significant difference in the neuronal activity in the two conditions during the selection interval (100-500 msec after the target dimmed). $B$ shows the same traces for the eight possible targets condition. The data are taken from trials in which the monkeys performed the task correctly. These neurons show a clear decline in activity associated with the identification of the target and the initiation of the saccade when they are in the preferred field and not when in the opposite hemifield. Horizontal calibration: $200 \mathrm{msec}$.

\section{Adjacent target locations}

In the different stimulus configurations, animals also made saccades to targets adjacent to the preferred location and to those in the hemifield opposite the preferred location. The activity profile of SNr neurons in the presence of eight possible targets affords a novel opportunity to examine a recent proposal regarding the role of the BG in the selection of preferred movements (Mink and Thatch, 1993; Mink, 1996). According to this scheme, decreases in activity of BG inhibitory output neurons act to disinhibit thalamocortical pathways responsible for generating a preferred movement, and increases act to inhibit thalamocortical pathways involved in producing the nonselected movement, movements that would compete with or interfere with the selected movement. Therefore, we measured the changes in activity of the SNr neurons when each of the eight stimuli in the eight stimulus conditions became the target for the saccade task. Of course this 

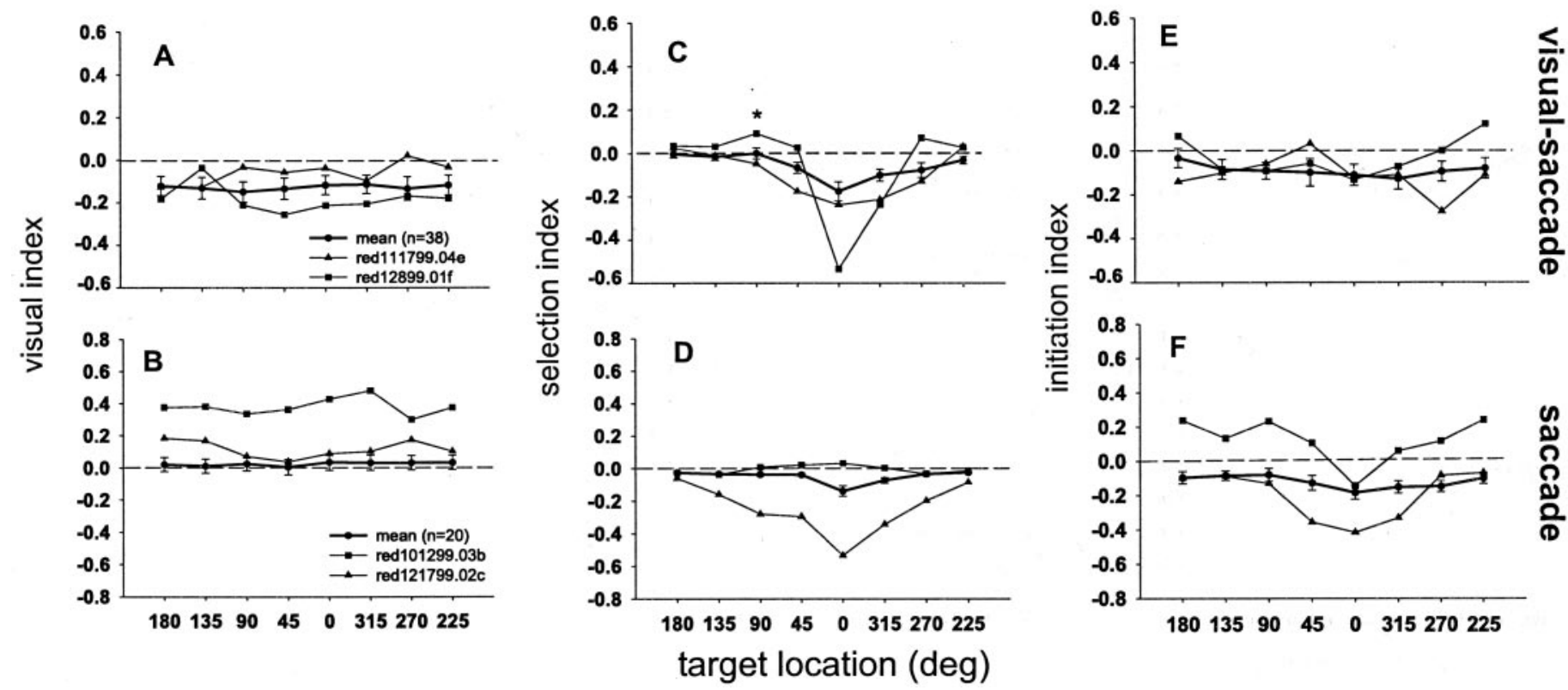

Figure 9. Selectivity indices for SNr visual-saccade neurons and $\mathrm{SNr}$ saccade neurons. Neuronal activity is plotted as a function of target direction. The activity is normalized to the $0^{\circ}$ location as the best response of the neurons in the eight possible targets condition. $A, B$, The visual index shows the activity of the neurons during the presentation of the visual stimulus 100-300 msec after onset of the array minus the $200 \mathrm{msec}$ of baseline activity (during fixation but before the array appeared) divided by the sum of the same two activities. $C, D$, The selection index was defined as the activity 400 msec after the target dimmed (beginning at $100 \mathrm{msec}$ after the dim) minus the $400 \mathrm{msec}$ period before the target dimmed divided by the sum of these two activities. $E, F$, The initiation index was defined as the $150 \mathrm{msec}$ around the saccade onset (before and after) minus the 200 msec baseline activity divided by the sum of the same two activities. In each plot, the results for two example neurons are shown $(\boldsymbol{\Lambda}, \mathbf{\square})$ as well as the mean of all neurons $(\bullet)$. Error bars indicate 1 SEM. The asterisk indicates that the increase was significantly greater than baseline (Mann-Whitney $U ; p<0.014$ ).

analysis was limited to $45^{\circ}$ resolution, because this was the minimum angle between the stimuli in our display. Nevertheless, it allowed us to compare lateral interactions, if any, within the $\mathrm{SNr}$ as we did in the SC and as has been done by Schall and colleagues (Schall and Hanes, 1993; Schall, 1995; Schall et al., 1995; Schall and Thompson, 1999) in frontal eye fields.

For both visual-saccade and saccade neurons of the $\mathrm{SNr}$, we calculated an index, essentially a contrast ratio, at three intervals of the task: visual, the time around the onset of the visual stimuli; selection, the time around the dimming of the target; and initiation, the time around saccade onset (Fig. 9). Figure 9 is arranged so that the preferred response of the neurons in the eight possible targets condition is rotated to the $0^{\circ}$ location.

We calculated the visual selectivity index by dividing the difference of the activity 100-300 msec after stimulus onset and the $200 \mathrm{msec}$ of baseline activity (during fixation before the array appeared) by the sum of the activity in the same two intervals (Fig. $9 A, B)$. A value of 0 indicates no difference in activity from baseline, values below 0 indicate that the activity is less than baseline, and values $>0$ indicate that the activity is greater than baseline. For the visual-saccade neurons, the initial activity was below baseline for the 2 example neurons (Fig. $9 A, \mathbf{\Delta}, \mathbf{\square}$ ) as well as the sample of 38 neurons (Fig. 9A, ). This is consistent with the pause in activity after visual stimulus onset, and also, because this interval occurred before the target was identified, the activity does not distinguish between targets or distractors, i.e., they are not tuned.

In contrast, for some saccade neurons, the initial visual activity increased when eight possible stimuli were presented (Fig. 9B, $\mathbf{\square}$, A) but also did not discriminate between targets and distractors. Although an increase in activity was evident in some neurons and across the sample initially (Fig. 6), it did not persist for our measurement interval across the sample (Fig. 9B, $\mathbf{0}$ ).
The contrast ratio calculated for the selection index was the difference between the $400 \mathrm{msec}$ before the target was identified and the $400 \mathrm{msec}$ of activity after the target was identified (beginning $100 \mathrm{msec}$ after the target dimmed) divided by the sum of the two activities (Fig. 9C,D). Some visual-saccade neurons were very broadly tuned during this interval (Fig. 9C, $\mathbf{\Delta}$ ), whereas others were less broadly tuned and showed a slight increase in activity only when a target adjacent to the preferred location was identified (Fig. 9C, $\mathbf{\square}$ ). Indeed, the increase at the $90^{\circ}$ location was significantly different from baseline (Mann-Whitney; ${ }^{*} p<0.014$ ). Although opposite in sign, this pattern is reminiscent of that seen in frontal eye fields (Schall and Hanes, 1993; Schall, 1995; Schall et al., 1995) and SC (Basso and Wurtz, 1998). Across the sample of 38 visual-saccade $\mathrm{SNr}$ neurons, there was a decline in activity associated with the preferred target location and less of a decline for adjacent targets.

Some saccade neurons showed a decline in activity at the time the target was identified that was broadly tuned for target location (Fig. 9D, $\mathbf{\Delta}$ ). More commonly, however, saccade neurons showed only a modest decline in activity at the time the target was identified (Fig. 9D, ○).

The contrast ratio for the initiation index was calculated by measuring the difference between the $100 \mathrm{msec}$ interval before and after saccade onset $(50 \mathrm{msec}$ before saccade and $50 \mathrm{msec}$ after saccade) and the baseline activity (200 msec before array onset during fixation). This difference was then divided by the sum of the two activities (Fig. 9E, F). Visual-saccade neurons of the $\mathrm{SNr}$ were not at all tuned during the initiation period but exhibited a level of activity that was reduced from baseline (Fig. $9 E, \mathbf{0})$.

For saccade neurons, around the time of saccade initiation, the neuron that showed broad tuning during the selection period 
showed a more restricted selectivity (Fig. $9 F, \mathbf{\Delta}$ ). A neuron that had negligible modulation during the selection period showed a decline for the preferred saccade location and an enhancement for all other saccade locations (Fig. 9F, $\square$ ). Because of the variability across our sample of saccade neurons, there was very little obvious tuning during the initiation period (Fig. $9 F, \mathbf{0}$ ).

In summary, there is a decline in activity for the preferred target location, with a lesser decline at adjacent target locations. Interestingly, there is a hint that visual-saccade neurons may increase their activity for target locations identified adjacent to the preferred location as if contributing to the suppression of distracter information. Perhaps this result would be more prominent if our stimuli were placed closer than $45^{\circ}$ from one another.

\section{Saccade latency}

Because SNr neurons are thought to have a role in the control of saccade initiation (Hikosaka and Wurtz, 1983d) and the delay period activity of SC neurons is associated with saccade latency in certain tasks (Dorris et al., 1997; Basso and Wurtz, 1998), we compared delay activity and saccade latency. We measured the $300 \mathrm{msec}$ of neuronal activity before the cue to move for all 58 neurons recorded and the saccade latency and calculated the Pearson $r$ value (Fig. 10). We found significant correlations for 4 of the 58 neurons, 1 of which was in the opposite direction (the activity decreased as saccade latency increased). In a few cases, activity preceding saccade generation of SNr neurons was a good predictor of saccade latency in this task. This is consistent with findings in SC (Dorris et al., 1997; Basso and Wurtz, 1998). We also compared saccade latency as a function of the number of possible saccade targets and found no significant differences. This probably results from the insertion of a delay (800-1200 msec) between the target identification and the onset of the cue to move that would obscure any latency differences between conditions as we saw previously (Basso and Wurtz, 1998).

\section{DISCUSSION}

We found a series of changes in SNr neuronal activity during the series of phases of our multitarget selection task. In a set of neurons that we classified as visual-saccade neurons, there was a pause in activity after the initial onset of the visual stimulus array and before the onset of the saccade as has been reported previously (Hikosaka and Wurtz, 1983a). In addition, we found that the activity after stimulus onset but preceding identification of the target remained below baseline. When the target was identified, there was a sharp, further decline in activity. This decline in activity was not just the result of the luminance change of the target but rather predicted which target the monkey would select as the goal of a saccade, because the decline was not present when the monkey made a mistake and made a saccade to a location other than the one indicated by the cue. The decline for the preferred target location was accompanied by a lesser decline for adjacent locations. Of these activity changes, only the pause after the onset of the array of stimuli was modulated by the number of possible targets. Most neurons that paused only with saccade onset showed no modulation related to selecting the visual target.

\section{Contribution of SNr to SC during target selection}

Because we used the same behavioral paradigm for the $\mathrm{SNr}$ as we had used previously for the SC (Basso and Wurtz, 1997, 1998), we can make a detailed comparison between the SNr and SC. In the SNr neurons that we have studied, the neuronal response during our multitarget selection task takes the form of a decrease in
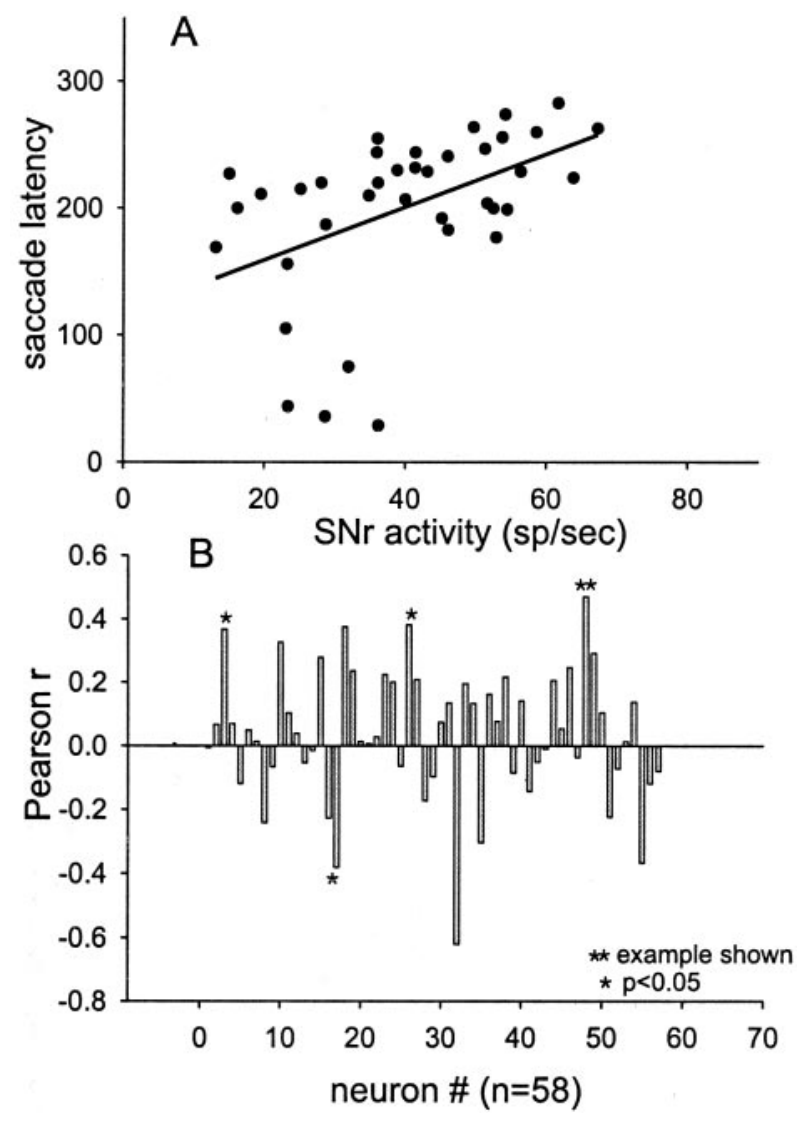

Figure 10. Correlation of saccade latency with $\mathrm{SNr}$ neuronal activity. $A$, Saccade latency as a function of neuronal activity averaged over $300 \mathrm{msec}$ before the cue to move in a single $\mathrm{SNr}$ neuron is plotted. The line is the regression through the data points. For this neuron, the Pearson $r$ value was 0.36 , which was statistically significant $(p<0.05)$. $B$, The distribution of $r$ values calculated for the 58 neurons. Three other $\mathrm{SNr}$ neurons had significant correlations with saccade latency (*), and the example in $A$ is indicated (**). Note that the data are taken from all four possible target conditions, and there are not four "clumps" of data points indicating that there were no differences in latency between the different target conditions.

activity, whereas in the SC the change is an increase in activity. Because the SNr projects to the SC (Hopkins and Niessen, 1976; Deniau et al., 1978; Graybiel, 1978; Anderson and Yoshida, 1980; Beckstead, 1983; Hikosaka and Wurtz, 1983d) and is inhibitory (Chevalier et al., 1981, 1984, 1985; Karabelas and Moschovakis, 1985), we can now consider the extent to which the decrease in $\mathrm{SNr}$ activity contributes to the increase in the SC during target selection. To facilitate this comparison, we show in Figure 11 the averaged activity of SC buildup neurons in the multitarget task that we reported previously (Basso and Wurtz, 1998, their Fig. 4) along with the averaged activity of the visual-saccade neurons from $\mathrm{SNr}$ (Fig. 5).

\section{Visual activity}

The initial visual pause of SNr neurons became less as the number of possible stimuli increased (Fig. 11B). This change with increasing numbers of stimuli could be caused either by the decreasing certainty that a given stimulus would be the target for a saccade or by the lateral interactions resulting from the decreasing distances between pools of neurons activated by the stimuli, or both. In our previous experiments on the SC, we concluded that the SC visual response was influenced by stimulus interac- 

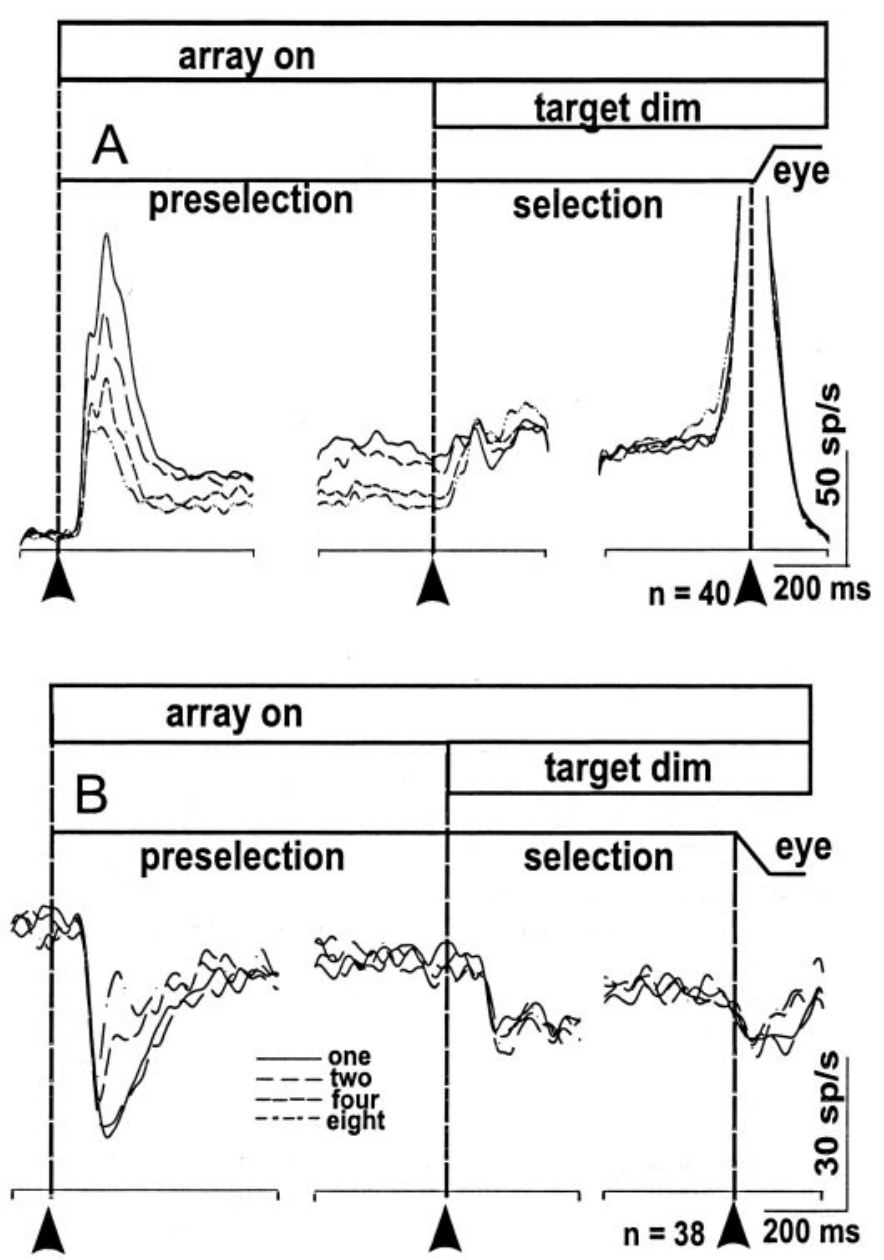

Figure 11. Comparison of SC and SNr neuronal activity in the multitarget task. $A$, The activity profiles of $40 \mathrm{SC}$ neurons recorded in the multitarget task. The spike density functions were averaged for each neuron and superimposed for the four stimulus conditions. Each plot is aligned as in the other figures. The left plot is aligned on the array onset, the middle plot is aligned on the target dim, and the right plot is aligned on saccade initiation. This figure was taken from Basso and Wurtz (1998). B, The arrangement of this plot is identical to the plot in $A$ except the data come from $\mathrm{SNr}$ recordings (see also Fig. 5). Note that the data from the $\mathrm{SC}$ and the $\mathrm{SNr}$ were recorded at different times and from different monkeys.

tions (Basso and Wurtz, 1998, their Fig. 11) as well as the certainty of the target location, on the basis of results from additional experiments. Moreover, it seemed likely that the change in target location certainty played a prominent role in the $\mathrm{SC}$, because moving from one stimulus to two was accompanied by a change in the amplitude of the visual response. This indicated either an effect of the change in certainty or long-range stimulus interactions, because the second stimulus was always in the opposite visual field. In the SNr, by contrast, addition of a second stimulus in the opposite visual field (reducing certainty by $50 \%$ ) had little effect on the visual response on average. The reduction became evident only with the addition of more stimuli. We think this suggests a larger role for close-range stimulus interactions in the SNr. The relative roles of target certainty and stimulus interactions will have to be determined in subsequent experiments.

Regardless of the nature of the visual response in the $\mathrm{SNr}$ and $\mathrm{SC}$, can the change in SNr account for the change in SC? As we have noted, with the addition of a second stimulus, SC activity changes but $\mathrm{SNr}$ activity does not. In addition, the latency of the visual response in the $\mathrm{SNr}$ is slightly longer than in the $\mathrm{SC}$, and so the modulation of the visual pause seen in the $\mathrm{SNr}$ cannot be responsible for the visual response modulations seen in the SC. This difference in latency was noted previously (Hikosaka and Wurtz, 1983a) and is evident by comparing the response profiles of the two classes of neuron during the array presentation (Fig. $11 A, B$, left plots). Therefore, although the $\mathrm{SNr}$ could contribute to the later SC visual response, it clearly cannot be the only input determining it.

\section{Delay activity}

In the preselection period after the response to the visual stimulus onset, the SNr activity remains below the baseline level and then declines further in the selection period after the target is identified. These changes are the same regardless of the number of stimuli. In the SC, the activity in the preselection period decreases as the number of possible targets increases. When the target is identified, the SC activity increases to levels seen in the single target condition regardless of the number of stimuli. Thus the change of activity at the start of the selection period is the same in SNr and SC (and of opposite sign), and the activity level reached in both is independent of the number of possible targets, that is, of the probability of a stimulus becoming a target. What is different is that the preselection activity in the SC is higher with fewer possible targets, whereas in the $\mathrm{SNr}$ it is not.

Because the preselection delay period activities were not perfectly matched in the SNr and SC, it is unlikely that SC modulations are a direct result of changes arising from the SNr. Rather, either the SNr activity seen during this time does not influence the $\mathrm{SC}$ activity or the changes seen in the $\mathrm{SNr}$ are indirectly responsible for changes in SC activity. For example, the reduced activity during the delay period may exert a permissive disinhibition across the SC map (Basso and Evinger, 1996; Basso et al., 1996; Schicatano et al., 1997), allowing cortical inputs or intrinsic SC interactions to directly regulate the level of SC buildup activity.

In contrast, in the selection period, the $\mathrm{SNr}$ could contribute to the SC activity, but only beyond the initial SC activity change, because the decline in activity in the $\mathrm{SNr}$ had a slightly longer latency than did the increase in SC, which is similar to the latency differences in the initial visual response. Examination of the middle plot of Figure 11 reveals that in the SC, there is initially an increase and then there appears an additional slight increase. It is possible that the initial, short-latency increase is caused by the luminance change in the preferred field that is not evident in $\mathrm{SNr}$ neurons (Fig. 11A,B, compare middle plots). Thus the decline in the $\mathrm{SNr}$ may be responsible for the longer latency increase in SC neurons in the selection period after the target is identified. Furthermore, the decline in the $\mathrm{SNr}$ activity at the time of target selection is clearly related to the saccade that is to be made rather than just to the target identified, because on error trials when the target was identified but the monkey did not go to it, the decline in $\mathrm{SNr}$ activity was absent. Unfortunately, there were too few error trials in the SC experiments to make a quantitative comparison.

What then is the influence of SNr on SC during target selection? When the target is identified on a given trial, the increase in the SC could result primarily from the release from inhibition from the $\mathrm{SNr}$ as the activity of the visual-saccade neurons declines. Such a coupling would be consistent with the timing of the 
inversely related $\mathrm{SNr}$ and SC activity. This activity is tied to the saccade that will be made (at least in the $\mathrm{SNr}$ ) and therefore is consistent with the original observations on the close relationship of the pause of activity in the $\mathrm{SNr}$ and the burst of activity in the SC associated with saccade initiation (Hikosaka and Wurtz, 1983d). We suggest that our observations are most consistent with the SNr making a substantial contribution to the SC activity as the target is being selected and as the saccade to that target is being generated.

\section{Saccade activity}

In the $\mathrm{SNr}$ visual-saccade neurons, the pause associated with the saccade was unmodulated with increases in the number of possible targets. Furthermore, across the sample, the $\mathrm{SNr}$ saccade neurons showed no modulation during any period in our task (Fig. 6). This behavior is consistent with a role for these neurons exclusively in the initiation of saccadic eye movements (Hikosaka and Wurtz, 1983c,d; Handel and Glimcher, 1999) and is consistent with our previous findings in the SC saccade-related activity of both burst and buildup neurons. Importantly, it is consistent with a substantial contribution of the $\mathrm{SNr}$ to the saccadic burst activity within the SC.

We conclude that in our multitarget selection task, a major, direct contribution of the SNr to SC activity is at the time of target selection and subsequently in the generation of the saccade to the selected target. Although the $\mathrm{SNr}$ may play a permissive role during the time after the stimulus onset and before the target is indicated, the modulation in SC seen with a different number of possible stimuli is likely dependent on intrinsic SC mechanisms or direct input from other sources or both.

\section{SNr spatial interaction and behavioral selection in the basal ganglia}

Our multitarget probability task also provided some information on the spatial extent of the modulations of $\mathrm{SNr}$ activity. A mechanism based on lateral interactions for saccade target selection has been proposed in frontal eye field (Schall and Hanes, 1993; Schall, 1995; Schall et al., 1995), and a similar mechanism has been proposed for movement selection in the BG (Mink and Thatch, 1993; Mink, 1996). This model of BG function emphasizes the role of the $\mathrm{BG}$ in the inhibition of unwanted movements and a selective disinhibition for wanted movements. This model was developed, in large part, to explain results obtained from recent anatomical work (Hazrati and Parent, 1992a,b; Parent and Hazrati, 1993) and reversible inactivation experiments of the globus pallidus (Mink and Thatch, 1991c).

Our experiments offered the opportunity to study such interactions in the saccadic system that are comparable to those of Mink and Thach (1993) in the skeletal motor system if we can regard a saccade to one part of the visual field to be the movement that is facilitated and the saccades to other parts of the field as those not facilitated. The original experiments of Hikosaka and Wurtz (1983d) showed that this is the case for the pause in activity associated with saccadic eye movements made to visual and remembered targets in one region of the visual field. Our current experiments show that at the time of target selection, the most prominent response was a pause for the preferred location and no change for the rest. Within the SC we had previously found similar patterns, albeit of opposite sign. In a few $\mathrm{SNr}$ neurons there was an indication that they increased their activity when stimuli adjacent to the preferred location were identified as targets (Figs. 9C,F), and some saccade neurons of the $\mathrm{SNr}$ exhib- ited an increase when many stimuli were present (Fig. 9B). We take this as providing some evidence for specificity of the lateral interactions within the BG. In our task, it is important to remember that there was a $45^{\circ}$ separation between stimuli, and given the observation that the $\mathrm{SNr}$ did not exhibit remote stimulus effects (no reduction in pause for two compared with one stimulus) (Fig. 5 ), the frequency of this finding may depend on the proximity of distracting stimuli to the target. Indeed, this may increase the likelihood of observing this phenomenon in SC and frontal eye field as well. Future experiments are required to map the resolution of these interactive effects.

In conclusion, the multitarget probability task shows that there is a pause in activity of the SNr neurons with target selection and saccade initiation to one region of the field, and this pause is limited to one part of the field. Taken together, these are probably the best evidence so far within the BG that there is a selective facilitation (via reduced inhibition) of chosen eye movements before their execution.

Note added in proof. Identification of the substantia nigra through the thalamus was described originally by Schultz (1986).

\section{REFERENCES}

Anderson ME, Yoshida M (1977) Electrophysiological evidence for branching nigral projections to the thalamus and the superior colliculus. Brain Res 137:361-375.

Anderson ME, Yoshida M (1980) Axonal branching patterns and location of nigrothalamic and nigrocollicular neurons in the cat. J Neurophysiol 43:883-895.

Basso MA, Evinger C (1996) An explanation for reflex blink hyperexcitability in Parkinson's disease. II. Nucleus raphe magnus. J Neurosci 16:7318-7330.

Basso MA, Wurtz RH (1997) Modulation of neuronal activity by target uncertainty. Nature 389:66-69.

Basso MA, Wurtz RH (1998) Modulation of neuronal activity in superior colliculus by changes in target probability. J Neurosci 18:7519-7534.

Basso MA, Powers AS, Evinger C (1996) An explanation for reflex blink hyperexcitability in Parkinson's disease. I. Superior colliculus. J Neurosci 16:7308-7317.

Beckstead RM (1983) Long collateral branches of substantia nigra pars reticulata axons to thalamus, superior colliculus and reticular formation in monkey and cat. Multiple retrograde neuronal labeling with fluorescent dyes. Neuroscience 10:767-779.

Boussaoud D, Desimone R, Ungerleider LG (1992) Subcortical connections of visual areas MST and FST in macaques. Vis Neurosci 9:291-302.

Bravo MJ, Nakayama K (1992) The role of attention in different visualsearch tasks. Percept Psychophys 51:465-472.

Britten KH, Shadlen MN, Newsome WT, Movshon JA (1992) The analysis of visual motion: a comparison of neuronal and psychophysical performance. J Neurosci 12:4745-4765.

Chandra R, Optican LM (1997) Detection, classification, and superposition resolution of action potentials in multiunit single-channel recordings by an on-line real-time neural network. IEEE Trans Biomed Eng 44:403-412.

Chevalier G, Deniau JM, Thierry AM, Feger J (1981) The nigro-tectal pathway. An electrophysiological reinvestigation in the rat. Brain Res 213:253-263.

Chevalier G, Vacher S, Deniau JM (1984) Inhibitory nigral influence on tectospinal neurons, a possible implication of basal ganglia in orienting behavior. Exp Brain Res 53:320-326.

Chevalier G, Vacher S, Deniau JM, Desban M (1985) Disinhibition as a basic process in the expression of striatal functions. I. The striato-nigral influence on tecto-spino/tecto-diencephalic neurons. Brain Res 334:215-226.

Cooper JR, Bloom FE, Roth RH (1986) The biochemical basis of neuropharmacology, Ed 5. Oxford: Oxford University Press.

Crist CF, Yamasaki DSG, Komatsu H, Wurtz RH (1988) A grid system and a microsyringe for single cell recording. J Neurosci Methods 26:117-122.

Deniau JM, Hammond C, Riszk A, Feger J (1978) Electrophysiological properties of identified output neurons of the rat substantia nigra (pars compacta and pars reticulata): evidences for the existence of branched neurons. Exp Brain Res 32:409-422.

Dorris MC, Munoz DP (1998) Saccadic probability influences motor preparation signals and time to saccadic initiation. $J$ Neurosci 18:7015-7026. 
Dorris MC, Paré M, Munoz DP (1997) Neuronal activity in monkey superior colliculus related to the initiation of saccadic eye movements. J Neurosci 17:8566-8579.

Fuchs AF, Robinson DA (1966) A method for measuring horizontal and vertical eye movement chronically in the monkey. J Appl Physiol 21:1068-1070.

Fuller JH, Schlag JD (1976) Determination of antidromic excitation by the collision test: problems of interpretation. Brain Res 112:283-298.

Glimcher PW, Sparks DL (1992) Movement selection in advance of action in the superior colliculus. Nature 355:542-545.

Graybiel AM (1978) Organization of the nigrotectal connection: an experimental tracer study in the cat. Brain Res 143:339-348.

Handel A, Glimcher PW (1999) Quantitative analysis of substantia nigra pars reticulata activity during a visually guided saccade task. J Neurophysiol 82:3458-3475.

Handel A, Glimcher PW (2000) Contextual modulation of substantia nigra pars reticulata neurons. J Neurophysiol 83:3042-3048.

Hays AV, Richmond BJ, Optican LM (1982) A UNIX-based multiple process system for real-time data acquisition and control. WESCON Conf Proc 2:1-10.

Hazrati L-N, Parent A (1992a) Convergence of subthalamic and striatal efferents at pallidal level in primates: an anterograde double-labelling study with biocytin and PHAL-L. Brain Res 569:336-340.

Hazrati L-N, Parent A (1992b) The striatopallidal projection displays a high degree of anatomical specificity in primate. Brain Res 592:213-227.

Hikosaka O, Wurtz RH (1983a) Visual and oculomotor functions of monkey substantia nigra pars reticulata. I. Relation of visual and auditory responses to saccades. J Neurophysiol 49:1230-1253.

Hikosaka O, Wurtz RH (1983b) Visual and oculomotor functions of monkey substantia nigra pars reticulata. II. Visual responses related to fixation of gaze. J Neurophysiol 49:1254-1267.

Hikosaka O, Wurtz RH (1983c) Visual and oculomotor functions of monkey substantia nigra pars reticulata. III. Memory-contingent visual and saccade responses. J Neurophysiol 49:1268-1284.

Hikosaka O, Wurtz RH (1983d) Visual and oculomotor functions of monkey substantia nigra pars reticulata. IV. Relation of substantia nigra to superior colliculus. J Neurophysiol 49:1285-1301.

Hopkins DA, Niessen LW (1976) Substantia nigra projections to the reticular formation, superior colliculus and central gray in the rat, cat, and monkey. Neurosci Lett 2:253-259.

Judge SJ, Richmond BJ, Chu FC (1980) Implantation of magnetic search coils for measurement of eye position: an improved method. Vision Res 20:535-538.

Karabelas AB, Moschovakis AK (1985) Nigral inhibitory termination on efferent neurons of the superior colliculus: an intracellular horseradish peroxidase study in the cat. J Comp Neurol 239:309-329.

Lemon R (1984) Methods for neuronal recording in conscious animals. In: IBRO Handbook Series: methods in the neurosciences, Vol 4, pp 95-102. New York: Wiley.
Ma TP (1996) Saccade-related omnivectoral pause neurons in the primate zona incerta. NeuroReport 7:2713-2716.

Mink JW (1996) The basal ganglia: focused selection and inhibition of competing motor programs. Prog Neurobiol 50:381-425.

Mink JW, Thatch WT (1991a) Basal ganglia motor control I. Nonexclusive relation of pallidal discharge to five movement modes. J Neurophysiol 65:273-300.

Mink JW, Thatch WT (1991b) Basal ganglia motor control II. Late pallidal timing relative to movement onset and inconsistent coding of movement parameters. J Neurophysiol 65:301-329.

Mink JW, Thatch WT (1991c) Basal ganglia motor control III. Pallidal ablation: normal reaction time, muscle cocontraction, and slow movement. J Neurophysiol 65:330-351.

Mink JW, Thatch WT (1993) Basal ganglia intrinsic circuits and their role in behavior. Curr Opin Neurobiol 3:950-957.

Munoz DP, Wurtz RH (1995) Saccade-related activity in monkey superior colliculus. I. Characteristics of burst and buildup cells. J Neurophysiol 73:2313-2333.

Parent A, Hazrati L-N (1993) Anatomical aspects of information processing in primate basal ganglia. Trends Neurosci 16:111-116.

Parthasarathy HB, Schall JD, Graybiel AM (1992) Distributed but convergent ordering of corticostriatal projections: analysis of frontal eye field and supplementary eye field in the macaque monkey. J Neurosci 12:4468-4488.

Schall JD (1995) Neural basis of saccade target selection. Rev Neurosci 6:63-85.

Schall JD, Hanes DP (1993) Neural basis of saccade target selection in frontal eye field during visual search. Nature 366:467-469.

Schall JD, Thompson KG (1999) Neural selection and control of visually guided eye movements. Annu Rev Neurosci 22:241-259.

Schall JD, Hanes DP, Thompson KG, King DJ (1995) Saccade target selection in frontal eye field of macaque. I. Visual and premovement activation. J Neurosci 15:6905-6918.

Schicatano EJ, Basso MA, Evinger C (1997) An animal model explains the origins of cranial dystonia benign essential blepharospasm. J Neurophysiol 77:2842-2846.

Schultz W (1986) Responses of midbrain dopamine neurons to behavioral trigger stimuli in the monkey. J Neurophysiol 56:1439-1462.

Selemon LD, Goldman-Rakic PS (1985) Longitudinal topography and interdigitation of corticostriatal projections in the rhesus monkey. J Neurosci 5:776-794.

Selemon LD, Goldman-Rakic PS (1988) Common cortical and subcortical targets of the dorsolateral prefrontal and posterior parietal cortices in the rhesus monkey: evidence for a distributed neural network subserving spatially guided behavior. J Neurosci 8:4049-4068.

Thompson KG, Hanes DP, Bichot NP, Schall JD (1996) Perceptual and motor processing stages identified in the activity of macaque frontal eye field neurons during visual search. J Neurophysiol 76:4040-4054.

Treisman AM, Gelade G (1980) A feature-integration theory of attention. Cognit Psychol 12:97-136. 